\title{
Analyse de la réponse au choc thermique chez les Streptomyces : caractérisation de protéines chaperons et détection de modifications post-traductionnelles thermo-dépendantes
}

\author{
P Mazodier 1, G Guglielmi 1, P Servant 1, \\ C Thompson 1, J Davies 2
}

\footnotetext{
1 Unité de génie microbiologique, Institut Pasteur, 28, rue du Dr Roux, Paris 75015, France;

${ }^{2}$ Department of Microbiology, University of British Columbia, Vancouver, BC V6T 1Z3, Canada
}

\begin{abstract}
Résumé - Afin de déterminer le rôle des heat shock proteins (HSP) dans certains des processus originaux propres aux streptomycètes (production d'antibiotiques, différenciation, sporulation), nous avons étudié la réponse à un choc thermique chez ceux-ci. Chez tous les streptomycètes étudiés 2 gènes de type groEL ont été mis en évidence. D'autre part nous avons montré que 3 protéines de type GroEL HSP18, HSP56 et HSP58 sont codées par ces 2 gènes chez $S$ albus. Le produit du gène groEL1 est à $30^{\circ} \mathrm{C}$ une protéine de $58 \mathrm{kDa}$ (HSP58), mais à haute température, une partie des messagers est traduite en une protéine écourtée de $18 \mathrm{kDa}$ (HSP18). HSP18 est également modifiée par l'adjonction d'un groupement chimique original. La modification post-traductionnelle semble être un phénomène général des protéines GroEL. L'étude immunologique suggère en effet qu'il y a une grande variation dans le caractère immunitaire des 3 protéines et que certains des épitopes reconnus sont dus à des modifications post-traductionnelles, thermo-dépendantes.
\end{abstract}

choc thermique / chaperon / GroEL / modification post-traductionnelle / Streptomyces albus

Summary - Survey of the heat-shock response in Streptomyces. Characterization of chaperonins and detection of temperature dependent post-translational modification. Exposure of living cells to heat shock results in dramatic changes in their pattern of protein synthesis. Some of the proteins induced are strongly conserved in the course of evolution this is the case for the molecular chaperon GroEL. In spite of the critical and diverse functions of the GroEL protein only one groEL gene has been reported in the numerous eubacteria that have been studied. However at least 2 groEL-like genes were present in all the Streptomyces species we tested. In Streptomyces albus the 2 groEL like genes groEL1 and groEL2 encode 3 proteins HSP56, HSP58 and an unusual HSP18. HSP58 and HSP18 which correspond to the N-terminal part of HSP58 are encoded by groEL1. Biochemical and immunological studies of HSP18 indicate a posttranslational modification of HSP18. Furthermore temperature-dependent posttranslational modification of GroEL and "GroEL like" proteins could be a common feature in bacteria; some of those modifications could be detected with monoclonal antibodies. 


\section{INTRODUCTION}

II peut paraître étonnant de trouver dans Le Lait un article traitant des streptomycètes; toutefois plusieurs arguments peuvent le justifier; certes, les Streptomyces ne sont pas des bactéries lactiques mais des eubactéries Gram positives de l'ordre des actinomycètes. Ils sont phylogénétiquement assez proches des Propionibacterium dont on connaît le rôle prépondérant dans l'élaboration des fromages à pâtes pressées cuites. L'étude génétique de ces dernières bactéries ne fait que commencer et peut profiter des résultats acquis chez d'autres actinomycètes. Cela est particulièrement vrai dans le domaine de la réponse au choc thermique, où l'étude des actinomycètes fait apparaître une situation profondément différente de celle établie chez Escherichia coli.

L'étude de la réponse au choc thermique et la caractérisation des protéines associées nous semblent particulièrement pertinentes pour l'industrie fromagère où les problèmes de culture mésophile sont quotidiens. En outre, les protéines de choc thermique de type chaperon ont des rôles universels. Rappelons que la protéine GroEL est impliquée dans la synthèse de la tête et de la queue de certains phages, et que la première mutation dans le gène groEL a historiquement été caractérisée chez E coli comme conférant la résistance au phage $\lambda$. Nous n'épiloguerons pas sur l'importance de la résistance phagique et ses implications dans l'industrie laitière.

\section{MÉTHODES}

Ces dernières années une nouvelle classe de protéines essentielles à la vie de la cellule a été mise en évidence : "les protéines chaperons" (Ellis, 1987; Hemmingsen et al, 1988). Ces "chaperonines", dont les paradigmes sont les protéines DnaK et GroEL de $E$ coli (voir Lindquist et Craig, 1988, pour une revue) sont impliquées dans divers processus fondamentaux impliquant des interactions protéine-protéine. Citons notamment la formation de complexes protéiques multimériques (Flynn et al, 1989), certaines translocations à travers des membranes (Deshaies et al, 1988; Kumamoto, 1991), le repliement (Laminet et al, 1990) et la renaturation de certaines protéines (Van Dyk et al, 1989). Plusieurs utilisations en biotechnologie de ces chaperons ont d'ores et déjà été développées. Citons chez les streptomycètes la récente utilisation de la protéine GroEL de $E$ coli pour la renaturation de polypeptides isolés par électrophorèse préparative sur gel de polyacrylamide dénaturant (Brown et al, 1992).

Un stress cellulaire comme une élévation brutale de la température (heat shock) suffit pour induire la synthèse de telles protéines, qui sont généralement synthétisées à un niveau modéré durant la croissance normale.

Les streptomycètes possèdent un cycle de différenciation morphologique complexe et produisent de très nombreux antibiotiques, d'où leur grand intérẽt industriel. Afin de déterminer l'implication éventuelle des heat shock proteins (HSP) dans certains des processus originaux propres aux streptomycètes (production d'antibiotiques, différenciation, sporulation), nous avons étudié la réponse à un choc thermique chez ceux-ci.

Lorsque nous avons commencé cette étude, la nature et la régulation de la réponse au choc thermique chez les streptomycètes étaient inconnues. Toutefois chez les mycobactéries, autres actinomycètes, des protéines de choc thermique avaient èté caractérisées. Ces dernières ont suscitè un intérêt considérable et de nombreuses spéculations depuis qu'il a été démontré qu'elles constituaient les antigènes dominants lors de l'infection ou de la vaccination (Shinnick, 1987 pour une revue, voir Young et al, 1988). Dans cet article, nous montrons que les streptomycètes présentent 3 protéines de type GroEL sujettes à des modifications posttraductionnelles oniginales, et qu'il y a une grande variation dans le caractère immunitaire de ces protéines. 


\section{RÉSULTATS}

\section{Réponse des streptomycètes à un choc thermique : détection de 2 gènes groEL}

La réponse à un choc thermique (passage rapide d'une culture de 30 à $37^{\circ} \mathrm{C}$ ) a été étudiée avec 15 souches de streptomycètes. Quatre d'entre elles (Streptomyces albus, $S$ lividans, $S$ parvulus, $S$ viridochromogenes) ont été sélectionnées pour des études plus approfondies : gels à une ou 2 dimensions après marquage des protéines totales par la méthionine $\left.{ }^{35} \mathrm{~S}\right]$ avant et après un choc thermique.

Dans toutes ces souches, 4 HSP majeures ont été observées; 3 correspondent aux HSP majeures de procaryotes Lon, DnaK et GroEL, la quatrième est une protéine plus petite ( 16 à $20 \mathrm{kDa}$ selon les espèces).

La taille et la régulation de la synthèse de cette protéine étaient originales. Nous avons choisi de l'étudier chez $S$ albus, où son niveau de synthèse est considérable (fig 1).

Chez $S$ albus, cette petite protéine de $18 \mathrm{kDa}$, absente à $30^{\circ} \mathrm{C}$, est fortement synthétisée après un choc thermique. De plus, sa synthèse reste à un niveau appréciable lorsque l'organisme pousse en continu à haute température : cette synthèse constitutive suggérait que le promoteur n'était pas sous la dépendance d'un facteur de transcription fugace, tel qu'un facteur sigma comparable au facteur 632 qui contrôle la transcription des gènes du régulon heat-shock chez E coli (Grossman et al, 1984, 1987).

HSP18 a été purifiée et partiellement séquencée par dégradation de Edman. La comparaison des séquences obtenues avec les banques de données a mis clairement en évidence une forte homologie de HSP18 avec l'extrémité NH2-terminale des protéines chaperons de type GroEL. Grâce aux données acquises, des oligonucléotides synthétiques ainsi que la partie $5^{\prime}$ du gène de la protéine de $65 \mathrm{kDa}$ de $\mathrm{Myco}-$ bacterium leprae ont été utilisés comme sondes dans des hybridations avec l'ADN génomique de $S$ albus. Deux gènes groEL like ont été mis en évidence (Guglielmi et al, 1991). Nous avons convenu d'appeler ces gènes groEL1 et groEL2.

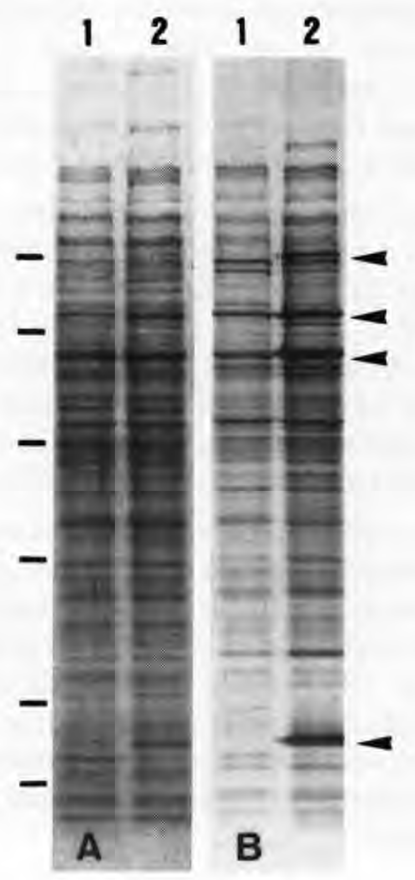

Fig 1. Réponse de $S$ albus à un choc thermique. Des marquages à la [35S] méthionine ont été effectués pendant $30 \mathrm{~min}$ sur une culture à $30{ }^{\circ} \mathrm{C}$ (ligne 1) ou immédiatement après passage de la culture de 30 à $37^{\circ} \mathrm{C}$ (ligne 2). Les extraits bruts réalisés ont été analysés par électrophorèse sur gel de SDS polyacrylamide. Les protéines ont été révélées par coloration au bleu de Coomassie (A) ou par autoradiographie (B). Les tirets correspondent à une gamme de marqueurs de $94,67,43,30,20,1$ et $14,4 \mathrm{kDa}$. Les flèches signalent de haut en bas les protéines Lon, DnaK, groEL et HSP18. 
La présence d'une famille de tels gènes, n'avait jamais été décrite chez les procaryotes et semblait jusqu'alors être une exclusivité des eucaryotes. Nous avons montré par hybridation que la présence de 2 gènes groEL-like était un phénomène commun à tous les Streptomyces étudiés (15 sur 15).

Chez $S$ albus, les 2 gènes groEL1 et groEL2 qui, sur le chromosome, sont très éloignés l'un de l'autre, ont été clonés par l'intermédiaire de cosmides, et séquencés (Mazodier et al, 1991). Seul le gène groEL1 est précédé d'un gène homologue au gène heat shock groES, situation comparable à celle observée chez E coli.

Les séquences nucléotidiques des 2 gènes groEL présentent $70 \%$ d'identité. Le produit du gène groEL2 contient à son extrémité $\mathrm{C}$ terminale le motif Gly Gly Met. Ce motif est présent à cette position dans toutes les séquences des protéines GroEL like publiées à ce jour à l'exception de la rubisco binding protein de chloroplaste.

Le produit du gène groEL1 en revanche ne présente pas ce motif, mais possède une extrémité $\mathrm{COOH}$ tout à fait originale et remarquable par sa richesse en histidine: Gly His Gly His Gly His Ser His Stop qui pourrait représenter un signal non encore déterminé. (Rappelons qu'une série d'histidines permet la fixation d'un métal comme le nickel).

\section{Trois protéines GroEL-like sont codées par 2 gènes groEL-like chez $\mathrm{S}$ albus}

Nous avons montré par purification des protéines et détermination des séquences $\mathrm{NH}_{2}$ terminales que les 2 gènes groEL sont transcrits et traduits (Guglielmi et al, 1991).

Le produit du gène groEL1 à $30^{\circ} \mathrm{C}$ est une protéine HSP58 dont la taille $58 \mathrm{kDa}$ et la séquence correspondent en première analyse à celles prédites à partir de la séquence nucléotidique (56 681 Da). A haute température, une partie des messagers de l'opéron groES-groEL1 est traduite en une protéine écourtée de $18 \mathrm{kDa}$ (HSP18). La présence de boucles putatives dans la région du gène groEL1, proche du lieu de terminaison de HSP18, pourrait suggérer un changement de phase de lecture du ribosome au cours de la traduction entraînant une terminaison prématurée. De plus, HSP18 semble fortement modifiée par un composé contenant du soufre par un processus original. En effet HSP18 est considérablement plus marquée par la [ ${ }^{35}$ ] méthionine que par la $\left[{ }^{14} \mathrm{C}\right]$ alanine $(6,9 \%$ du marquage totale des protéines contre $3,8 \%$ ) alors que la séquence prédite pour HSP18 contient peu de méthionines et beaucoup d'alanines.

Par ailleurs, après marquage par la [35S] méthionine, puis purification et coupure de HSP18 au bromure de cyanogène, il reste 2 peptides marqués alors que le soufre des méthionines impliquées dans la chaîne peptidique est éliminé sous forme de méthylthiocyanate. Une partie du soufre [ ${ }^{35}$ S] présent dans la protéine HSP18 marquée ne se trouve donc pas dans les méthionines impliquées dans les liaisons peptidiques.

Le produit du gène groEL2 est une protéine de $56 \mathrm{kDa}$ (HSP56) qui correspond à la taille que l'on peut prédire à partir de la séquence nucléotidique (56 $682 \mathrm{Da})$. Ces résultats sont résumés sur la figure 2 .

\section{Détection de modifications thermo- inductibles dans les protéines GroEL}

L'analyse en gel à 2 dimensions, des extraits bruts protéiques de $S$ albus réalisés à partir d'une culture à $30^{\circ} \mathrm{C}$, montre plusieurs taches adjacentes pouvant correspondre à diverses modifications des pro- 
téines HSP56 et HSP58 (fig 3). Dans les extraits faits après choc thermique, on observe l'apparition d'au moins 2 nouvelles taches correspondant à des protéines d'environ $56 \mathrm{kDa}$ avec des points isoélectriques plus acides (Guglielmi et al, 1991).

II est tentant d'interpréter ce résultat dans le contexte de l'observation de Sherman et Goldberg (1992). Ces auteurs ont récemment montré chez E coli qu'une fraction des protéines GroEL était phosphorylée en condition de choc thermique.

Les nombreuses taches observées après électrophorèse en 2 dimensions peuvent être interprétées comme des formes modifiées des protéines HSP56 et HSP58.

Afin de déterminer la nature des différentes taches, une étude immunologique avec des anticorps anti GroEL a été entreprise.

Les préparations d'anticorps polyclonaux de lapin anti GroEL de $E$ coli que nous avons utilisées, reconnaissent en Western Blot le produit de groEL2 (HSP56) mais ne reconnaissent ni HSP18, ni HSP58, bien que les 2 protéines HSP56 et HSP58 présentent le même pourcen-

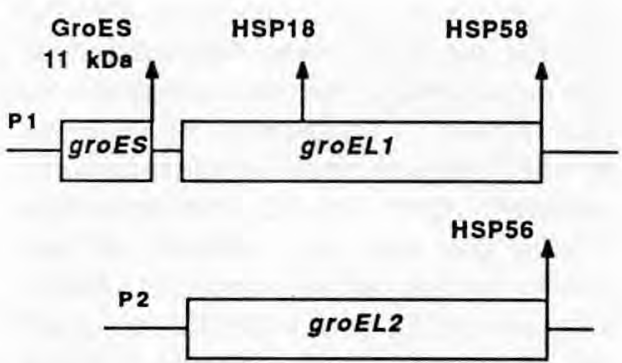

Fig 2. Représentation schématique des gènes groE de $S$ albus et de leurs produits.

tage d'identité avec la protéine GroEL de $E$ coli $(54 \%)$.

Ce fait n'est peut-être pas sans précédent. En effet depuis que nous avons reporté pour la première fois chez un procaryote la présence de 2 gènes groEL-like, cette même observation a été refaite dans le genre Mycobacterium (Rinke de Wit et al, 1992 et communication personnelle des Drs Coates et Kong). Ces auteurs ont en effet détecté et cloné un second gène groEL-like respectivement chez Mycobacterium leprae et $M$ tuberculosis. Ceci est
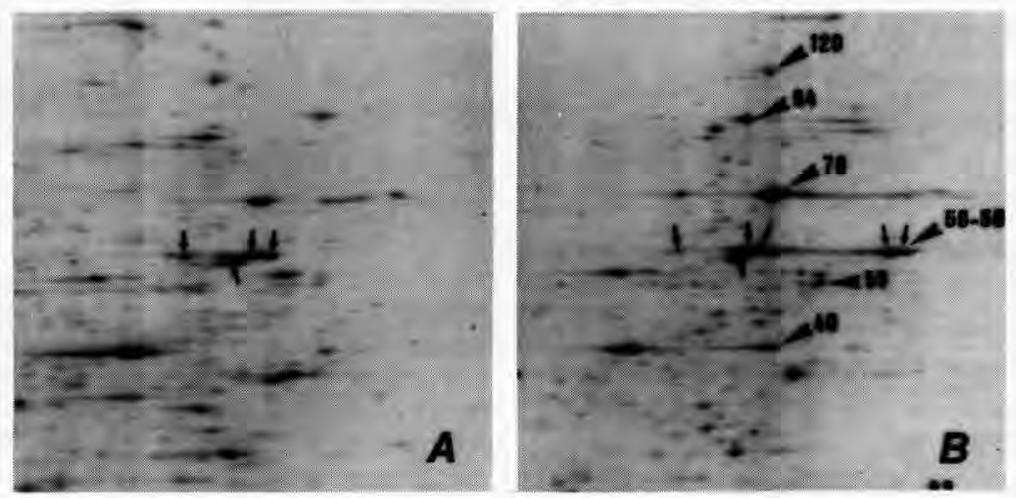

Fig 3. Analyse en gel à 2 dimensions de la réponse au choc thermique chez $S$ albus. Les cultures ont été marquées pendant $20 \mathrm{~min}$ avec de la [ $\left.{ }^{35} \mathrm{~S}\right]$ méthionine à $30^{\circ} \mathrm{C}$ (A) ou immédiatement après passage à $42^{\circ} \mathrm{C}(\mathrm{B})$. Les cônes indiquent les protéines de choc thermique, les petites flèches indiquent les produits possibles des gènes groEL. 
très surprenant car "l'antigène de 65 kDa" (produit de groEL chez Mycobacterium) est certainement l'un des antigènes les plus étudiés. "L'antigène de $65 \mathrm{kDa}$ " est en effet habituellement l'antigène immunodominant dans les 2 pathogénicités. D'autre part plus de 3 milliards de personnes ont été vaccinés contre la tuberculose avec le BCG et le produit de groEL est également majoritairement reconnu après ces immunisations, tant au niveau sérologique que par sa capacité à stimuler les lymphocytes $T$. Des banques d'anticorps monoclonaux reconnaissant divers épitopes de l'antigène de $65 \mathrm{kDa}$ ont été établies au cours de programmes OMS visant à caractériser les antigènes majeurs de $M$ tuberculosis, du BCG et de $M$ leprae. Si la situation chez Mycobacterium est celle que nous avons décrite chez les streptomycètes, c'est-à-dire que les deux gènes groEL-like sont exprimés, nous nous retrouverions, chez Mycobacterium aussi, avec le paradoxe de l'absence de détection immunologique de l'une des 2 protéines GroEL.

Par ailleurs, un lapin a été immunisé avec de la protéine HSP18 purifiée. Les anticorps obtenus reconnaissent en Western Blot la protéine HSP18 mais non la protéine HSP58 (fig 4).

Ces résultats sont également paradoxaux, puisque HSP18 correspond à la partie $\mathrm{NH} 2$ terminale de HSP58. Plusieurs explications sont possibles :

Les épitopes accessibles sur HSP18 pourraient être masqués sur HSP58 dans les conditions expérimentales, mais cela est peu vraisemblable, les protéines étant dénaturées et déroulées lors de la migration en SDS-PAGE; ou bien les déterminants communs à HSP18 et HSP58 sont faiblement immunogènes par rapport aux épitopes spécifiques de HSP18 : nouvelle séquence peptidique dans la région $\mathrm{C}$ terminale due à un changement du cadre de lecture (frame shift) ou groupement modificateur ajouté post-traductionnellement.

D'autres résultats suggèrent également que des groupements ajoutés posttraductionnellement sur GroEL peuvent entraîner la création d'épitopes majeurs. La figure 5 montre que l'anticorps monoclonal "YI.2" dirigé contre la protéine GroEL de $M$ leprae reconnaît chez $S$ albus une protéine (HSP57 ?) dont le profil de migration

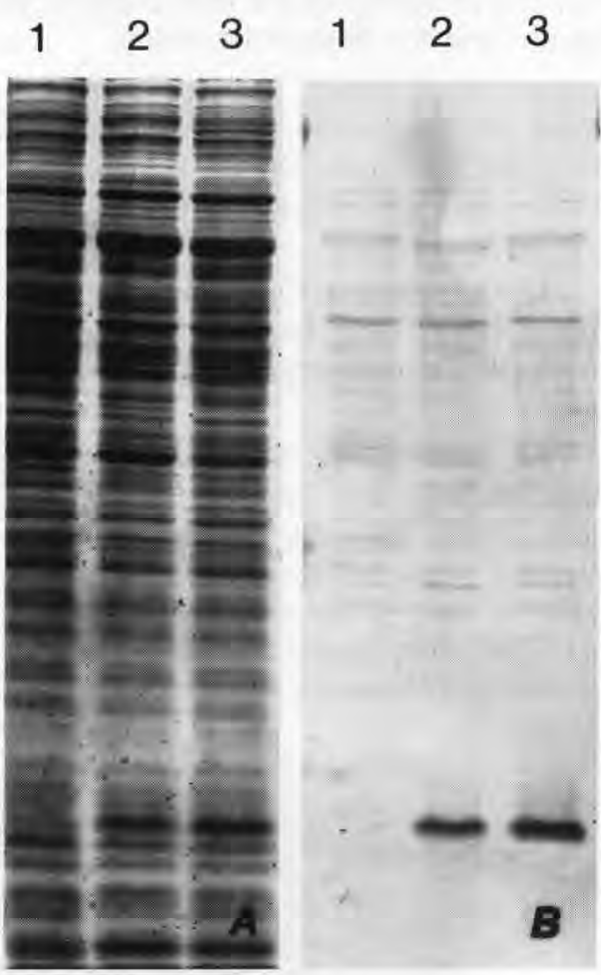

Fig 4. Utilisation des anticorps polyclonaux antiHSP18. Des extraits bruts ont été faits après : culture de $S$ albus à $30^{\circ} \mathrm{C}$ (ligne 1 ); $18 \mathrm{~h}$ de culture à $37^{\circ} \mathrm{C}$ (ligne 2); $40 \mathrm{~min}$ après passage de la culture de 30 à $37^{\circ} \mathrm{C}$ (ligne 3). Les extraits ont été soumis à une séparation par SDS-PAGE $(12,5 \%$ acrylamide) transférés à une membrane d'immobilon. La détection a été faite avec des anticorps polyclonaux de lapin anti-HSP18 (purifiés par affinité avec de l'HSP18). La révélation est faite avec des anti-immunoglobulines de lapin couplées à de la phosphatase. 
sur gel est intermédiaire entre ceux des protéines HSP56 et HSP58. Cette forme de la protéine est peu abondante à $30^{\circ} \mathrm{C}$, elle est davantage présente après choc thermique et s'accumule lors d'une culture à haute température $\left(37^{\circ} \mathrm{C}\right)$.

Les anticorps monoclonaux "Y1.2" reconnaissent-ils chez $S$ albus une éventuelle forme phosphorylée d'HSP56 ? Un

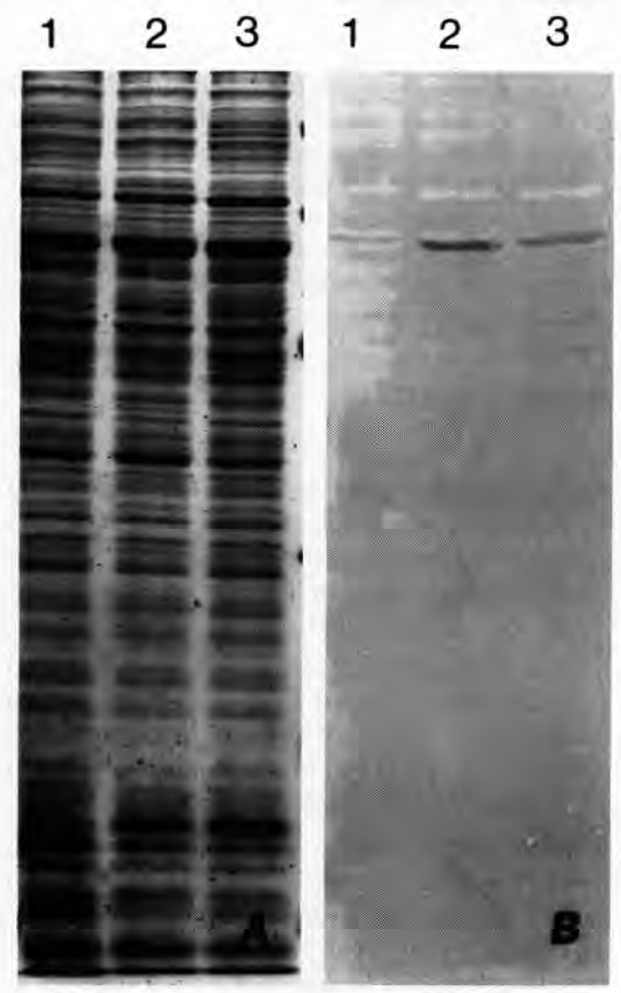

Fig 5. Détection d'une forme modifiée de GroEL chez $S$ albus après élévation de la température. Les extraits bruts ont été faits, traités et disposés de la même façon que sur la figure 4 . La détection a été faite avec la préparation d'anticorps monoclonaux "H105.10" (G Damiani, D Young, MRC Londres). Ceux-ci reconnaissent le même épitope situé dans la partie NH2 terminale de la protéine GroEL (65 kDa antigène) de $M$ leprąe que les anticorps monoclonaux " $Y 1.2$ ". La révélation est faite avec des anti-immunoglobulines de souris couplées à la phosphatase. traitement par de la phosphatase pourrait permettre de vérifier ce point.

Cependant cela semble a priori exclu; la figure 6 montre l'épitope reconnu par les anticorps monoclonaux "Y1.2" dans la protéine GroEL de $M$ leprae (Anderson et al, 1988) et les régions correspondantes dans les protéines HSP56 et HSP58 de $S$ albus. Les séquences de ces régions de GroEL chez $S$ albus ne présentent ni sérine, ni thréonine, ni tyrosine pouvant servir de site de phosphorylation.

Par ailleurs, les résultats obtenus par Anderson et al lors de la caractérisation des épitopes de GroEL chez M leprae peuvent être interprétés comme une indication que les anticorps "Y1.2" reconnaissent une modification de la protéine. Ces auteurs ont en effet montré que l'inhibition de la reconnaissance anticorps "Y1.2»/ protéine GroEL dans un test ELISA par des peptides synthétiques se faisait avec une faible efficacité.

Cet ensemble de résultats suggère donc que les anticorps monoclonaux "Y1.2" pourraient reconnaître un site de GroEL dont la modification est thermodépendante.

\section{DISCUSSION}

La détection et le clonage de 2 gènes de type groEL ainsi que la caractérisation de leurs 3 produits posent de nombreux problèmes théoriques quant aux rôles et aux modes de formation de ces produits. Ces résultats laissent aussi entrevoir certaines

$$
\begin{array}{ll}
\text { M. leprae } & \text { KTLAYDEEARRG ERGLNSLADAVK } \\
\text { S. albus GroEL2 } & \text { KILFDEEARRGLERGMNQLADAYK (21/25) } \\
\text { S. albus GroEL1 } & \text { KILKFDEDARRALERGVNQLADTYK (16/25) }
\end{array}
$$

Fig 6. Épitope reconnu par les anticorps monoclonaux Y1.2. 
applications. Ainsi le clonage de ces gènes fortement exprimés a rendu disponibles leurs promoteurs. Ceux-ci ont été utilisés avec succès pour l'expression de protéines hétérologues dans divers actinomycètes. L'expression d'une protéine virale sous contrôle de l'un de ces promoteurs chez un $M$ bovis BCG recombinant a permis d'induire une réponse immunitaire humorale et cellulaire contre cet antigène, une première étape dans l'obtention d'une souche vaccinale (Winter et al, 1991), La disponibilité des gènes groE a permis d'effectuer des expériences de mutagenèse dirigée in vivo par interruption de gènes (Servant et al, 1993).

Par ailleurs, la notion selon laquelle certains des épitopes présents sur plusieurs protéines GroEL, peuvent être dus à des modifications post-traductionnelles thermo-dépendantes va conduire à une reconsidération de certains des résultats établis en épidémiologie immuno-moléculaire dans les pathologies dues à $M$ tuberculosis et $M$ leprae.

De nombreuses questions posées par le paradoxe posé par 2 gènes codant pour 3 protéines restent sans réponse : quel est le mécanisme conduisant à HSP18 et quelle est la modification posttraductionnelle présente sur HSP18? Pour répondre à ces questions nous avons entrepris la purification d'une quantité importante d'HSP18 afin d'en déterminer la composition chimique et la séquence $\mathrm{C}$ terminale.

Quelles sont les modifications posttraductionnelles éventuellement présentes sur HSP56 ou HSP58 ? Celles-ci ont-elles une fonction dans le rôle de ces protéines?

Quel est le rôle de la protéine HSP18 ? L'étude biochimique de la protéine purifiée pourra nous donner des éléments de réponse.

Finalement, nous aimerions connaître la structure native des protéines GroEL chez $S$ albus. Chez E coli, GroEL est une protéine oligomérique (14 mers) constituée de 2 anneaux ( 7 mers) empilés. Quelle est la situation chez $S$ albus ? Y a-t-il présence de 2 homo-oligomères (HSP56) et (HSP58) ou d'hétéro-oligomères (HSP56/ HSP58), sous quelle forme se trouve HSP18?

\section{REMERCIEMENTS}

L'étude immunologique a été en grande partie rendue possible grâce aux anticorps polyclonaux et monoclonaux que nous a généreusement fournis le Dr D Young. Nous sommes également reconnaissants aux Drs Coates et Kong (St Georges Hospital, Londres) pour nous avoir communiqué leurs résultats avant publication.

\section{RÉFÉRENCES}

Anderson D, Barry M, Buchanan T (1988) Exact definition of species-specific and crossreactive epitopes of the 65-kilodaltons protein of Mycobacterium leprae using synthetic peptides. J Immunol 141, 607-613

Brown K, Wood S, Buttner M (1992) Isolation and characterization of the major vegetative RNA polymerase of Streptomyces coelicolor $A 3(2)$; renaturation of a sigma subunit using GroEL. Mol Microbiol 6, 1133-1139

Deshaies R, Koch B, Werner-Washburne M, Craig, E, Schekman R (1988) A subfamily of stress proteins facilitates translocation of secretory and mitochondrial precursor polypeptides. Nature 332, 800-805

Ellis R (1987) Proteins as molecular chaperones. Nature 328 , 378-379

Flynn G, Chappell T, Rothman J (1989) Peptide binding and release by proteins implicated as catalysts of protein assembly. Science 245, 385-390

Grossman A, Erickson J, Gross C (1984) The htpR gene product of $E$ coli is a sigma factor

- for heat-shock promoters. Cell 38, 383-390

Grossman A, Straus D, Walter W, Gross C (1987) $\sigma^{32}$ synthesis can regulate the synthe- 
sis of heat shock proteins in Escherichia coli. Genes Dev 1, 179-184

Guglielmi G, Mazodier P, Thompson C, Davies $J$ (1991) A survey of the heat shock response in four Streptomyces species reveals two groEL-like genes and three groEL-like proteins in Streptomyces albus. J Bacteriol 173, 7374-7381

Hemmingsen S, Woolford C, van der Vies S, Tilly K, Dennis D, Georgopoulos C, Hendrix R, Ellis R (1988) Homologous plant and bacterial proteins chaperone oligomeric protein assembly. Nature $333,330-334$

Kumamoto C (1991) Molecular chaperones and protein translocation across the Escherichia coli inner membrane. Mol Microbiol 5, 19-22

Laminet A, Ziegelhoffer T, Georgopoulos C, Plückthun A (1990) The Escherichia coli heat shock proteins GroEL and GroES modulate the folding of the $\beta$-lactamase precursor. EMBO J 9, 2315-2319

Lindquist S, Craig E (1988). The heat-shock proteins. Annu Rev Genet 22, 631-677

Mazodier P, Guglielmi G, Thompson J, Davies J (1991) Characterization of two groEL-like genes in Streptomyces albus. J Bacteriol $173,7382-7386$

Rinke de Wit T, Bekelie S, Osland A, Miko T, Hermans $P$, van Soolingen D, Drijfthout JW, Schöningh R, Janson A, Thole J (1992) Mycobacteria contain two groEL genes : the se- cond Mycobacterium leprae groEL gene is arranged in an operon with groES. Mol Microbiol 6,1995-2007

Servant P, Thompson C, Mazodier P (1993) Utilisation of new $E$ coli/Streptomyces conjugative vectors : inactivation of one of the two groEL-like genes of $S$ albus do not impair cell growth. Gene

Sherman M, Goldberg A (1992) Heat shock in Escherichia coli alters the protein-binding properties of the chaperonin groEL by inducing its phosphorylation. Nature $357,167-$ 169

Shinnick T (1987) The 65-kilodalton antigen of Mycobacterium tuberculosis. J Bacteriol 169, 1080-1088

Van Dyk T, Gatenby A, LaRossa R (1989). Demonstration by genetic suppression of interaction of GroE products with many proteins. Nature $342,451-453$

Winter N, Lagranderie M, Rauzier J, Timm J, Leclerc C, Guy B, Kieny M, Gheorgiu M, Gicquel B (1991) Expression of heterologous genes in Mycobacterium bovis BCG : induction of a cellular response against HIV-1 Nef protein. Gene 109, 47-54

Young D, Mehlert A, Bal V, Mendez-Samperio P, Ivanyi J, Lamb J (1988) Sress proteins and the immune response to mycobacteria. Antigens as virulence factors ? Antonie van Leeuwenhoek 54, 431-439 